\title{
Chromosomal Aberrations Induced by Retention of Cyprinid Fish Unfertilized Eggs in Freshwater
}

\author{
Takayoshi Ueda \\ Utsunomiya University, 350 Mine, Utsunomiya 321, Japan
}

Accepted October 21, 1996

The exploration for the factor of chromosomal changes would be the search for the factor of karyotype differentiation. Up to date, in fishes the chromosomal aberration caused by the formation of a hybrid, the temperature or the pressure shock during early development and the aged egg has been reported (Ueda and Aoki 1994, Ueda and Kobayashi 1988, Ueda et al. 1984, 1988, Yamazaki et al. 1989). Because it is known that the rates of fertilization and survival decrease in proportion as the time of retention of unfertilized eggs in freshwater gets longer, it is expected that the chromosomal aberration is induced by retention of eggs.

On the other hand, species facing extinction have been increasing remarkably in number, and it is urged that these species should be conserved. In bitterlings, four species, namely Acheilognathus longipinnis, Tanakia tanago, Rhodeus atremius suigensis and $R$. ocellatus kurumeus, were taken up as endangered species by the Environment Agency in Japan. In the near future, we may have to depend upon artificial fertilization in order to conserve these endangered species. I think it is important to make clear the influence upon the chromosome by retention of unfertilized eggs not only in the field of fisheries in which artificial fertilization is used frequently, but also in the field concerning in the conservation of species. The understanding of the relationship between the appearance of the chromosomal aberration and the retention of unfertilized eggs must be very important to make clear the mechanism of the occurrence of the chromosomal change and the karyotype differentiation.

In this study, chromosomes of embryos developed from bitterling eggs retained in the freshwater or the preservative solution before fertilization were analyzed.

\section{Materials and methods}

Six kinds of crossings from different parents in the rose bitterling (Rhodeus ocellatus ocellatus) were done (Groups A, B, C, D, E and F). In each group, eggs were obtained from 4-10 females and sperm were obtained from 1-2 males. An additional crossing of 3-5 female rose bitterlings and one male flat bitterling (Acheilognathus rhombeus) was done (Group G). Eggs were taken out and kept in $25^{\circ} \mathrm{C}$ freshwater (Groups A, B, C, D, E and G) or in $25^{\circ} \mathrm{C}$ preservative solution $\left(6.80 \mathrm{~g} \mathrm{NaCl}, 0.40 \mathrm{~g} \mathrm{KCl}, 0.20 \mathrm{~g} \mathrm{CaCl}_{2}, 0.20 \mathrm{~g} \mathrm{MgSO}_{4} \cdot 7 \mathrm{H}_{2} \mathrm{O}\right.$ and $3.57 \mathrm{~g}$ HEPES dissolved in 1 litter of the distilled water, adjusted to $\mathrm{pH} 8.2$ with $\mathrm{NaOH}$ ) (Group F). Eggs were fertilized at $0,5,10,15$ and 20 min after the taking out of eggs in Groups A and G, at 5, 10, 15, 20, 25 and $30 \mathrm{~min}$ in Groups B, C, D and E, at 0, 15, 30, 45, 60, 75, 90, 105 and $120 \mathrm{~min}$ in Group F. The fertilized eggs were kept at $20^{\circ} \mathrm{C}$ in the freshwater. The chromosome slides were made from embryos at around the gastrula stage (at about $20 \mathrm{hr}$ after fertilization) by the direct air-drying method with the conventional Giemsa stain (Ueda et al. 1991). Standard karyotypes of the rose bitterling and the flat bitterling were made from kidney cells by the direct method with the conventional Giemsa stain (Ueda et al. 1990). 


\section{Results}

As shown in Table 1, the percentage of gastruration had a tendency to decrease in proportion as the time of retention of eggs in freshwater or preservative solution got longer.

Fig. 1 shows the standard karyotype of the rose bitterling $(2 n, 48)$, consisting of 28 meta-

Table 1. Percentages of gastrulation developed from eggs retained in $25^{\circ} \mathrm{C}$ freshwater (Groups A, B,

$\mathrm{C}, \mathrm{D}, \mathrm{E}$ and $\mathrm{G})$ or preservative solution (Group F) before fertilization

\begin{tabular}{|c|c|c|c|}
\hline Group & $\begin{array}{l}\text { Retention } \\
\quad(\mathrm{min})\end{array}$ & $\begin{array}{l}\text { No. of } \\
\text { eggs }\end{array}$ & $\begin{array}{c}\text { Gastrulation } \\
(\%)\end{array}$ \\
\hline \multirow[t]{5}{*}{ A } & 0 & 20 & 100.0 \\
\hline & 5 & 20 & 100.0 \\
\hline & 10 & 20 & 80.0 \\
\hline & 15 & 20 & 35.0 \\
\hline & 20 & 20 & 10.0 \\
\hline \multirow[t]{6}{*}{ B } & 5 & 20 & 80.0 \\
\hline & 10 & 20 & 30.0 \\
\hline & 15 & 20 & 40.0 \\
\hline & 20 & 20 & 0.0 \\
\hline & 25 & 20 & 0.0 \\
\hline & 30 & 20 & 0.0 \\
\hline \multirow[t]{6}{*}{$\mathrm{C}$} & 5 & 21 & 95.2 \\
\hline & 10 & 19 & 36.8 \\
\hline & 15 & 21 & 4.8 \\
\hline & 20 & 20 & 0.0 \\
\hline & 25 & 21 & 0.0 \\
\hline & 30 & 20 & 0.0 \\
\hline \multirow[t]{6}{*}{ D } & 5 & 19 & 94.7 \\
\hline & 10 & 20 & 50.0 \\
\hline & 15 & 18 & 5.6 \\
\hline & 20 & 20 & 0.0 \\
\hline & 25 & 21 & 0.0 \\
\hline & 30 & 21 & 0.0 \\
\hline \multirow[t]{6}{*}{$\mathrm{E}$} & 5 & 23 & 52.0 \\
\hline & 10 & 21 & 0.0 \\
\hline & 15 & 19 & 0.0 \\
\hline & 20 & 21 & 0.0 \\
\hline & 25 & 20 & 0.0 \\
\hline & 30 & 18 & 0.0 \\
\hline \multirow[t]{9}{*}{$\mathrm{F}$} & 0 & 19 & 94.7 \\
\hline & 15 & 20 & 100.0 \\
\hline & 30 & 20 & 95.0 \\
\hline & 45 & 20 & 100.0 \\
\hline & 60 & 20 & 95.0 \\
\hline & 75 & 20 & 95.0 \\
\hline & 90 & 18 & 55.6 \\
\hline & 105 & 19 & 78.9 \\
\hline & 120 & 20 & 65.0 \\
\hline \multirow[t]{5}{*}{$\mathrm{G}$} & 0 & 20 & 100.0 \\
\hline & 5 & 20 & 95.0 \\
\hline & 10 & 21 & 81.0 \\
\hline & 15 & 21 & 38.1 \\
\hline & 20 & 25 & 28.0 \\
\hline
\end{tabular}

(M) and submetacentrics (SM), 20 subtelo(ST) and acrocentrics (A). This karyotype had one pair of the smallest ST-A with the satellite.

Table 2 shows the distribution of chromosomal numbers of embryos with chromosomal aberrations which were developed from eggs retained in the freshwater before fertilization (Groups A, B, D and E). But, results from embryos with normal karyotype are not included in this Table. The normal karyotype was obtained from following embryos: 3 embryos of $0 \mathrm{~min}$ retention, $3 \mathrm{em}$ bryos of $5 \mathrm{~min}$ retention, 5 embryos of $10 \mathrm{~min}$ retention, 3 embryos of $15 \mathrm{~min}$ retention in Group A; 4 embryos of 5 min retention, 2 embryos of $10 \mathrm{~min}$ retention, 3 embryos of 15 min retention in Group B; 5 embryos of 5 min retention, 5 embryos of 10 min retention in Group C; 5 embryos of 5 min retention, 3 embryos of 10 min retention in Group D; 6 embryos of 5 min retention in Group E. The mode of chromosomal numbers in Embryo A-1 was coincident with the normal number, but the karyotype with 48 chromosomes was different from the normal karyotype because it had a micro-chromosome. Both Embryos A-2 and B-1 were 47/48 mosaics, but the exact relationship of the chromosomal constitution between 47 and 48 could not be analyzed. All metaphase figures had a microchromosome in Embryo B-1. In Embryos A4, B-2, D-1 and E-1, the distribution of chromosomal numbers was wide and the mode was not clear. And, many microchromosomes were observed. In Embryo D1, metaphase figures with 24 chromosomes, with 25 chromosomes including a microchromosome, with 26 chromosomes including 2 micro-chromosomes and with 32 chromosomes including 8 micro-chromosomes were recognized. In Embryo A-4, structural aberrations of ring and dicentric chromosomes were conspicuous. In Group E, an 


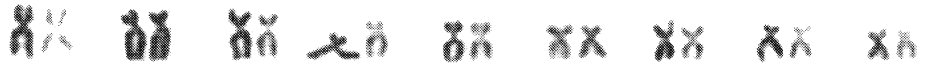

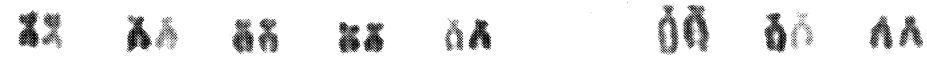

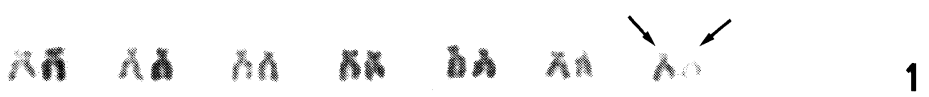

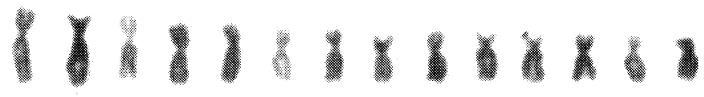

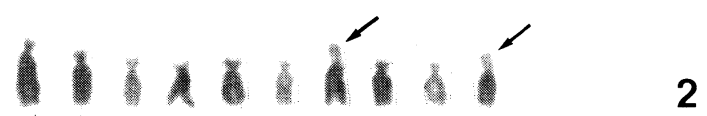 \\ 118118188088 \\ 1601018063

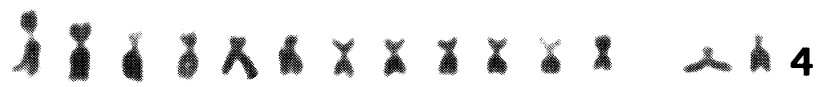

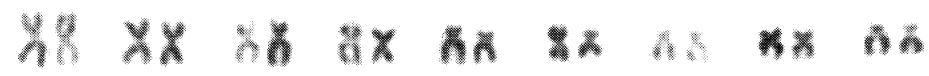

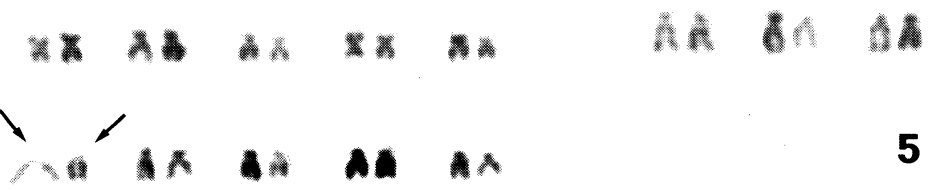

Figs. 1-5. 1. The standard karyotype of the rose bitterling $2 n=48$ (28 M-SM+20 ST-A). Arrows indicate one pair of the smallest ST-A with the satellite. 2. A karyotype with 24 chromosomes (14 M-SM+10 ST-A). Arrows indicate two ST-A with the satellite. 3. The haploid karyotype of the rose bitterling. $n=24(14 \mathrm{M}-\mathrm{SM}+10 \mathrm{ST}-\mathrm{A})$. An arrow indicates one smallest ST-A with the satellite. 4. A karyotype with 14 chromosomes (12 M-SM+2 ST-A). 5. The standard karyotype of the flat bitterling. $2 n=44$ (28 M-SM+16 ST-A). Arrows indicate 4 th pair of ST-A with the satellite.

embryo with chromosomal aberrations was developed from a egg retained even for $5 \mathrm{~min}$ in the freshwater, and no gastrulation was recognized in eggs retained for more than $10 \mathrm{~min}$.

All of 10 metaphases per one embryo had the standard karyotype of the rose bitterling in following embryos in Group F: 5 embryos of 0 min retention, 5 embryos of 15 min retention, 4 embryos of $30 \mathrm{~min}$ retention, 5 embryos of $45 \mathrm{~min}$ retention, 4 embryos of 60 min retention, 5 embryos of $75 \mathrm{~min}$ retention, 4 embryos of 90 min retention, 4 embryos of $105 \mathrm{~min}$ retention, 2 embryos of $120 \mathrm{~min}$ retention. Metaphase figures with structural chromosomal aberrations of the ring chromosome and the pulverization were observed at the rate of $30.8 \%$ in Embryo F1 and at the rate of $10.5 \%$ in Embryo F-5. All embryos developed from 0, 15, 45 and 75 min retention eggs had the normal karyotype $(2 n=48)$. Table 3 shows the distribution of 
Table 2. Distribution of chromosomal numbers in rose bitterling embryos derived from eggs retained in the freshwater before fertilization

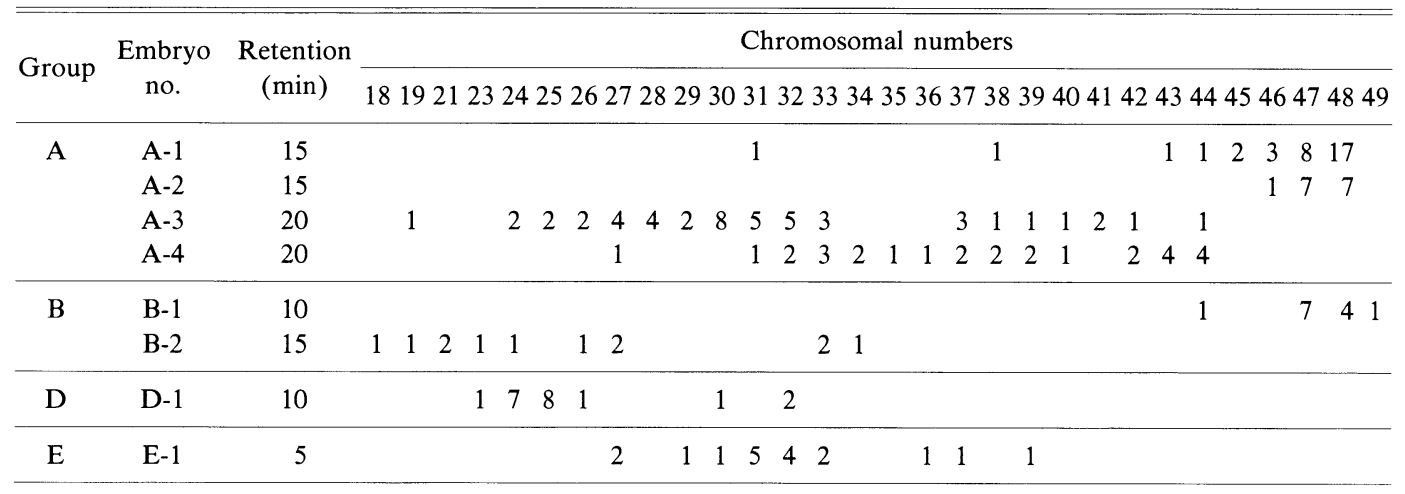

Table 3. Distribution of chromosomal numbers in rose bitterling embryos derived from eggs retained in the preservative solution before fertilization

\begin{tabular}{|c|c|c|c|c|c|c|c|c|c|c|c|c|c|c|c|c|c|c|c|c|c|c|c|c|c|c|}
\hline \multirow{2}{*}{ Group } & \multirow{2}{*}{$\begin{array}{c}\text { Embryo } \\
\text { no. }\end{array}$} & \multirow{2}{*}{$\begin{array}{l}\text { Retention } \\
\quad(\min )\end{array}$} & \multicolumn{24}{|c|}{ Chromosomal numbers } \\
\hline & & & 13 & 314 & 15 & 20 & 21 & 22 & 23 & 24 & 25 & 28 & 29 & 30 & 34 & 35 & 41 & 42 & 44 & 46 & 48 & 49 & 505 & 535 & 559 & 91 \\
\hline \multirow[t]{7}{*}{$\mathrm{F}$} & $\mathrm{F}-1$ & 30 & & & & & & 4 & 4 & 18 & & & & & & & & & & & & & & & & \\
\hline & $\mathrm{F}-2$ & 60 & & & 1 & 1 & 3 & 3 & & 3 & & & & & & & & & & & & & & & & \\
\hline & F-3 & 90 & & & & & & & & & & & & & & & & 3 & 1 & 1 & 3 & 1 & 2 & & & \\
\hline & F-4 & 105 & & & & & & & & & & 1 & & 1 & & & 1 & 1 & & 1 & & & & 1 & & \\
\hline & F-5 & 120 & 1 & 9 & 1 & & & & 3 & & 1 & 1 & 1 & & & & & & & & & & & & & \\
\hline & F-6 & 120 & & & & & & & & & & & & & 1 & 1 & & 1 & & & 4 & & & & 11 & 1 \\
\hline & F-7 & 120 & & & & & & & & 15 & & & & & & & & & & & & & & & & \\
\hline
\end{tabular}

Table 4. Distribution of chromosomal numbers in rose bitterling 우 $\times$ flat bitterling $\sigma^{7}$ embryos derived from eggs retained in the freshwater before fertilization

\begin{tabular}{|c|c|c|c|c|c|c|c|}
\hline \multirow{2}{*}{ Group } & \multirow{2}{*}{$\begin{array}{c}\text { Embryo } \\
\text { no. }\end{array}$} & \multirow{2}{*}{$\begin{array}{l}\text { Retention } \\
\quad(\min )\end{array}$} & \multicolumn{5}{|c|}{ Chromosomal numbers } \\
\hline & & & 242526 & 283 & 3743 & & 47 \\
\hline \multirow[t]{9}{*}{ G } & G-1 & 10 & 10 & & & & \\
\hline & G-2 & 10 & 10 & & & & \\
\hline & G-3 & 15 & 10 & & & & \\
\hline & G-4 & 15 & 10 & & & & \\
\hline & G-5 & 15 & $\begin{array}{lll}8 & 2 & 1\end{array}$ & 1 & & & \\
\hline & G-6 & 15 & & & 11 & 13 & 1 \\
\hline & G-7 & 20 & 10 & & & & \\
\hline & G-8 & 20 & 10 & & & & \\
\hline & G-9 & 20 & 10 & & & & \\
\hline
\end{tabular}

chromosomal numbers of embryos with chromosomal aberrations derived from eggs retained in the preservative solution before fertilization (Group F). But, results from embryos with normal karyotype are not included in this Table. In the group of 30 min retention, one embryo with the mode at 24 chromosomes was observed (Embryo F-1). Two metaphase figures with 24 chromosomes in Embryo F-1 had 14 M-SM and 10 ST-A (Fig. 2). This chromosomal constitution is the normal haploid type, but two chromosomes had the satellite, while the normal haploid has one. In groups of 60,90 and 105 min retention, three embryos had wide distri-

butions of chromosomal numbers (Embryos F-2, F-3 and F-4). The relationship of three karyotypes among metaphase figures with 42, 48 and 50 chromosomes in Embryo F-3 was not made clear. In the group of 120 min retention, 3 embryos had abnormal karyotypes. All of 15 metaphase figures in Embryo F-7 had 24 chromosomes. The karyotype of all of 5 metaphase figures were the normal haploid karyotype with $14 \mathrm{M}-\mathrm{SM}$ and $10 \mathrm{ST}$-A, including one smallest ST-A with the satellite (Fig. 3). Embryo F-6 had the mode at 48 chromosomal numbers, but showed a wide distribution. In Embryo F-5 with the wide distribution of chromosomal numbers, the mode was recognized at 14 chromosomal numbers. All of 9 metaphase figures 


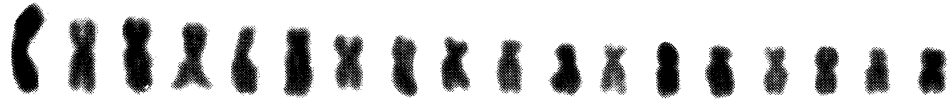

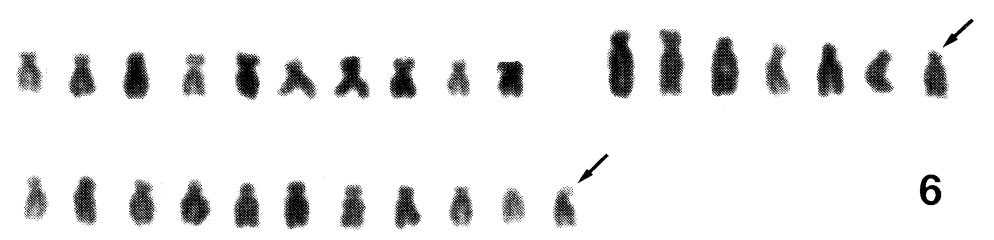

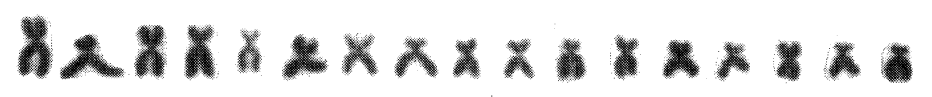

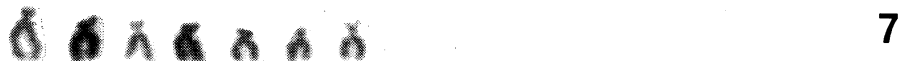

Figs. 6,7. 6. The standard intermediate karyotype between the rose bitterling and the flat bitterling with 46 chromosomes (28 M-SM+18 ST-A). Arrows indicate two ST-A with the satellite. 7. karyotype with 24 chromosomes (17 M-SM+7 ST-A).

with 14 chromosomes had 12 M-SM and 2 ST-A (Fig. 4).

Fig. 5 shows the normal karyotype of the flat bitterling $(2 n, 44)$, consisting of $28 \mathrm{M}-\mathrm{SM}+$ 16 ST-A. This karyotype had the satellite on the 4th pair of ST-A. All of 10 metaphases per one embryo had the normal intermediate karyotype between the parents, with $28 \mathrm{M}-\mathrm{SM}$ and 18 ST-A, in following embryos: 5 embryos of 0 min retention, 5 embryos of 5 min retention, 3 embryos of 10 min retention, 1 embryo of 15 min retention, 2 embryos of 20 min retention (Fig. 6). Metaphase figures with structural chromosomal aberrations were observed at the rate of 25.0\% in Embryo G-5 and at the rate of 33.3\% in Embryo G-6. Table 4 shows the distribution of chromosomal numbers of embryos with chromosomal aberrations derived from eggs retained in the freshwater before fertilization (Group G; rose bitterling 우 $\times$ flat bitterling $\sigma^{\nearrow}$ ). But, results from embryos with the normal karyotype are not included in this Table. In the group of 10 min retention, 2 embryos (G-1 and G-2) had 24 chromosomes which comprised 14 M-SM and 10 ST-A including one smallest ST-A with the satellite, and this karyotype was normal haploid of the rose bitterling. In the group of $15 \mathrm{~min}$ retention, 2 embryos with 24 chromosomes and 1 embryo with 46 chromosomes were observed. Embryo G-5 had the mode at 24 chromosomal numbers, but showed a wide distribution. Three metaphase figures with 24 chromosomes in this embryo had 17 M-SM and 7 ST-A, and this karyotype is quite different from the normal haploid type (Fig. 7). Two metaphase figures with 25 chromosomes had two different karyotypes: $17 \mathrm{M}-\mathrm{SM}+8$ ST-A and $15 \mathrm{M}-\mathrm{SM}+10 \mathrm{ST}-\mathrm{A}$. And also, many structural chromosomal aberrations were observed. The conspicuous chromosomal change was observed in Embryo G-6 with the mode at 46 chromosomal numbers, the wide distribution and many structural aberrations. In the group of $20 \mathrm{~min}$ retention, 3 embryos with 24 chromosomes and 2 embryos with 46 chromosomes were observed. Three embryos (G-7, G-8 and G-9) with 24 chromosomes, which comprised 14 M-SM and 10 ST-A including one smallest ST-A with the satellite, had normal haploids of the rose bitterling. Two embryos with 46 chromosomes, which comprised $28 \mathrm{M}-\mathrm{SM}$ and $18 \mathrm{ST}-\mathrm{A}$, including the 7 th and the smallest ST-A with the satellite, had intermediate-types between the parents. 


\section{Discussion}

I consider that the unfertilized egg retained in freshwater or preservative solution would be one of the aged eggs. It is known that the chromosomal aberration of polyploids and heteroploids is induced by the aging of eggs in mammals. The incidence of Down syndrome with no. 21 chromosome trisomy increases with the mother's age, as is well known. In amphibians, under the observation of trisomy, monosomy and mosaic embryos developed from overripe eggs retained in the follicle, it was shown that the aging of eggs induced the non-disjunction and the exclusion of chromosomes during the meiotic division and the cleavage (Witschi and Laguens 1963). There are only a few reports which have investigated the relationship between the chromosomal change and the aging of eggs in fishes. In Oncorhynchus mykiss and $O$. masou, the decrease of the fertilization rate, the increase of abnormal embryonic development and the increase of chromosomal aberrations including a high incidence of haploidy, hyperdiploidy and mosaicism in developing embryos, caused by prolonged retention of eggs in the coelomic cavity, have been recognized (Yamazaki et al. 1989). In this study, similar chromosomal aberrations to those described above were observed and the rate of gastrulation had a tendency to decrease in proportional as the time of retention of eggs got longer. It is presumed that the influence of retention is similar to that of aging of eggs. In Group E, an embryo had structural aberrations even in the 5 min retention group and the gastrulation was not recognized in eggs retained for more than $10 \mathrm{~min}$. I think that eggs used in Group $\mathrm{E}$ had already aged in the body. It is known that the artificial crossing with the low survival rate is frequently experienced in fish breeding. I consider the chromosomal aberration induced by the aging or retention of eggs as one of the main causes of the decrease in survival rate. This fact suggests the necessity of careful dealing in crossing for fish breeding, such as the certain conservation of original species. We need to take notice of the aging or retention of eggs also in the conservation of scare species.

There were many embryos with a wide distribution of chromosomal numbers and the indistinct mode. In these, mosaics which probably arose from unequal migration of chromosomes during cleavage were recognized. Structural chromosomal aberrations were observed in many gastrulas. These facts suggest that chromosomes had been changing violently while still at the gastrula stage. In a previous study of Xenopus (Witschi and Laguens 1963), abnormal embryos with the trisomy, the monosomy or the mosaic developed from eggs aged in the follicle were recognized, and it was shown that the aging of eggs induced the non-disjunction and the exclusion of chromosomes during meiotic division and cleavage. It is interesting that the aging of eggs may induce the abnormal chromosomal division of the non-disjunction not only at meiotic division but also at cleavage. It is presumed that the change of the component which lies within the cytoplasm, caused by the aging of eggs, has a harmful influence on the division and the structure of chromosomes during early development, or that the chromosomal change caused during meiotic division induces new changes at the following cell division. I cannot determine exactly why retention of unfertilized eggs induced the chromosomal aberrations in this study. But, there are some reports about the disorder of the formation of the spindle induced by the aging of eggs in mammals. The disorganization and the loss of spindle microtubles and chromosome displacement caused by the aging of eggs in mice were observed by Eichenlaub-Ritter et al. (1986). It may be considered that the chromosomal aberration in the present study is deeply related to the disorder of formation of the spindle.

Many haploid embryos developed from eggs retained in freshwater and the preservative solution were recognized. All haploid embryos (Group $G$ ) analyzed in rose bitterling o $\times$ flat bitterling $\sigma^{7}$ had only the maternal genome. I think haploid embryos in the present study were induced by retention of unfertilized eggs, because embryos derived from 8 previous routine 
artificial crossings from different parents of rose bitterling 우 $\times$ flat bitterling $\sigma^{7}$ had only an intermediate karyotype in my laboratory (unpublished data). In fishes, it has not been reported that certain gynogenetic haploids or diploids develope from aging eggs. Metaphase figures with 1-8 micro-chromosomes in Embryo D-1 may be derived from the haploid cell. Almost all haploids in the present study had the clean mode of chromosomal numbers. From these facts, it is presumed that these haploids did not result from the exclusion of the chromosome step by step, but were induced in a moment at the fertilization or the early cleavage.

A few embryos with the mode slipped from the normal chromosomal number were recognized. Embryo F-5 with 14 chromosomes is a conspicuous case. All of its metaphase figures had the same chromosomal constitution, and I can infer that this embryo settled down into a new karyotype. This fact suggests the reconstruction of the chromosome during development. Though Embryo F-1 had the mode of 24 chromosomal numbers, this karyotype included 2 chromosomes with the satellite. The constitution of 24 chromosomes in Embryo G4 was quite different from that of the normal haploid. Many structural chromosomal aberrations were observed in these embryos, too. Though we cannot explain clearly how these haploidies were induced, these observations may show the process of the reconstitution of the chromosomes.

I suppose that the appearance of embryos with chromosomal aberrations means the possibility of the karyotype differentiation. I have been considering the possibility of the karyotype differentiation, in which after chromosomes are changed during the early development, chromosomes are reconstructed with restoration or promotion during the increase and the differentiation of cells, and consequently a large scale of chromosomal change is induced in a short period (Ueda 1993, Ueda and Aoki 1994). I speculate that the disorder of chromosomal numbers and the appearance of structural chromosomal aberrations at around the gastrula stage show one of the expressions of this reconstruction.

\section{Summary}

Chromosomes of embryos developed from eggs of bitterling retained in the freshwater or the preservative solution before fertilization were analyzed. The percentage of gastrulation had a tendency to decrease, and an increase of chromosomal aberration was observed, in proportion as the time of retention of unfertilized eggs got longer. It is presumed that the retention of eggs is one of the main causes of the karyotype differentiation. Retention of eggs is necessary to be taken notice not only in the field of fisheries in which artificial fertilization is used frequently, but also in the field concerning the conservation of species.

Key words: Acheilognathine fishes, Retention of eggs in freshwater, Chromosomal aberration, Karyotype differentiation.

\section{Acknowledgments}

This research was supported by grants from the Fisheries Agency, Tokyo 100, Japan, and Japan Fisheries Resource Conservation Association, Tokyo 104, Japan.

\section{References}

Eichenlaur-Ritter, U., Chandley, A. C. and Gosden, R. G. 1986. Alterations to the microtubular cytoskelton and increased disorder of chromosome alignment in spontaneously ovulated mouse oocytes aged in vivo: an immunofluorescence study. Chromosoma 94: 337-345. 
Ueda, T. 1993. How do fish karyotypes change? Biological Science (Tokyo) 45: 182-186. (in Japanese)

- and Aoki, K. 1994. Chromosomal aberrations induced by cold shock in fertilized eggs of rose bitterling. Proc. of Fourth Indo-Pacific Fish Conference: 473-483.

-, Hayashi, M., Koide, N., Sofuni, T. and Kobayashi, J. 1991. Preliminary examination of the mutagenicity test using embryo cells of rose bitterling, Rhodeus ocellatus ocellatus. Chrom. Inf. Serv. 51: 12-14.

-, Kawakami, R. and Kobayashi, J. 1990. Rose bitterling as a laboratory animal for study of cell engineering and for teaching materials. Bull. Takaharayama Inst. Nature Educ. 9: 8-17. (in Japanese)

- and Kobayashi, J. 1988. Heteroploidy induced by production of triploids in the konanee, Oncorhynchus nerka. Chrom. Inf. Serv. 44: 7-8.

-, Ojima, Y., Sato, R. and Fukuda, Y. 1984. Triploid hybrids between female rainbow trout and male brook trout. Bull. Japan. Soc. Sci. Fish. 50: 1331-1336.

- Sato, R. and Kobayashi, J. 1988. Silver-banded karyotypes of the rainbow trout and the brook trout and their hybrids: Disappearance in allotriploids of Ag-NORs originated from the brook trout. Jpn. J. Genet. 63: 219226.

Witschi, E. and Laguens, R. 1963. Chromosomal aberrations in embryos from overripe eggs. Develop. Biol. 7: 605616.

Yamazaki, F., Goodier, J. and Yamano, K. 1989. Chromosomal aberrations caused by aging and hybridization in charr, masu salmon and related salmonids. Physiol. Ecol. Japan, Spec. Vol. 1: 529-542. 